\title{
Comparison of Time-Resolved and First-Pass Contrast- Enhanced MR Angiography in Pretherapeutic Evaluation of Spinal Dural Arteriovenous Fistulas
}

\author{
(D) S. Mathur, (D) A. Bharatha, (D)T.J. Huynh, DR.I. Aviv, and (DS.P. Symons
}

\begin{abstract}
BACKGROUND AND PURPOSE: Different MRA techniques used to evaluate spinal dural arteriovenous fistulas offer unique advantages and limitations with regards to temporal and spatial resolution. The purpose of this study was to compare the efficacy and interobserver agreement of 2 commonly used contrast-enhanced spinal MRA techniques, multiphase time-resolved MRA and single-phase first-pass MRA, in assessment of spinal dural arteriovenous fistulas.
\end{abstract}

MATERIALS AND METHODS: Retrospective review of 15 time-resolved and 31 first-pass MRA studies in patients with clinical suspicion of spinal dural arteriovenous fistula was performed by 2 independent, blinded observers. DSA was used as the reference standard to compare the diagnostic performance of the 2 techniques.

RESULTS: There were 10 cases of spinal dural arteriovenous fistula in the time-resolved MRA group and 20 in the first-pass MRA group. Time-resolved MRA detected spinal dural arteriovenous fistulas with sensitivity and specificity of $100 \%$ and $80 \%$, respectively, with $100 \%$ correct-level localization rate. First-pass MRA detected spinal dural arteriovenous fistulas with sensitivity and specificity of $100 \%$ and $82 \%$, respectively, with $87 \%$ correct-level localization rate. Interobserver agreement for localization was excellent for both techniques; however, it was higher for time-resolved MRA. In 5 cases, the site of fistula was not included in the FOV, but a prominent intradural radicular vein was observed at the edge of the FOV.

CONCLUSIONS: Multiphase time-resolved MRA and single-phase first-pass MRA were comparable in diagnosis and localization of spinal dural arteriovenous fistulas and demonstrated excellent interobserver agreement, though there were more instances of ambiguity in fistula localization on first-pass MRA.

ABBREVIATIONS: FP-MRA = first-pass MRA; SDAVF $=$ spinal dural arteriovenous fistula; TR-MRA $=$ time-resolved MRA

$\mathbf{S}$ pinal dural arteriovenous fistulas (SDAVFs) represent an abnormal connection between a radiculomeningeal artery and a radicular vein, typically on the dural sleeve adjacent to the nerve root. The arterialized radicular vein causes regurgitation of blood flow to the perimedullary venous plexus, resulting in increased venous pressure and congestion. ${ }^{1}$ Although SDAVFs are a treatable cause of myelopathy, ${ }^{2}$ the diagnosis remains challenging because the clinical and conventional MR imaging features are non-

Received June 30, 2016; accepted after revision August 12.

From the Division of Neuroradiology, Department of Medical Imaging (S.M., T.J.H., R.I.A., S.P.S.) and Department of Otolaryngology-Head and Neck Surgery (S.P.S.), Sunnybrook Health Sciences Centre, Toronto, Ontario, Canada; and Division of Diagnostic and Interventional Neuroradiology, Department of Medical Imaging (S.M., A.B., T.J.H.), St. Michael's Hospital, Toronto, Ontario, Canada.

Paper previously presented in part at: Annual Meeting of the American Society of Neuroradiology, April 25-30, 2015; Chicago, Illinois.

Please address correspondence to Sean P. Symons, MD, 2075 Bayview Ave, AG31D, Toronto, ON, Canada M4N 3M5; e-mail: sean.symons@sunnybrook.ca

http://dx.doi.org/10.3174/ajnr.A4962 specific. As a result, misdiagnosis and delay in diagnosis are common, which may result in additional disability. ${ }^{3}$

MRA is useful for confirming the diagnosis and for localizing SDAVFs to expedite DSA. ${ }^{4,5}$ DSA is ultimately the "criterion standard" for diagnosis of this condition, but the number of injections and the time required to perform this study can be reduced with the knowledge of the level involved, provided by MRA. The contrast-enhanced spinal MRA techniques useful for evaluation of SDAVFs are broadly of 2 types: first-pass and time-resolved. These differ with regards to temporal and spatial resolution. The purpose of this study was to compare the efficacy and interobserver agreement of multiphase time-resolved MRA (TR-MRA) and single-phase first-pass MRA (FP-MRA) in assessment of SDAVFs.

\section{MATERIALS AND METHODS Patient Cohort}

Research ethics review board approval was obtained for this retrospective study from both the Sunnybrook Health Sciences Cen- 
tre and St. Michael's Hospital. From January 2000 to April 2015, all patients who were referred to the medical imaging departments of the 2 participating sites with a clinical suspicion of SDAVF and underwent conventional spine MR imaging, contrast-enhanced TR-MRA or FP-MRA, and DSA were included in the study. Patients with a history of treated SDAVF were excluded. On retrospective review of imaging and clinical data bases, 58 patients were found to satisfy these criteria.

Patients found to have SDAVF variants on DSA including epidural AVF, perimedullary AVF, spinal cord AVM, and filum terminale AVF were excluded $(n=12)$ because they were unequally distributed in the TR-MRA and FP-MRA groups (1 and 11, respectively). This yielded a total of 15 and 31 patients in the TRMRA and FP-MRA groups, respectively.

\section{MR Imaging and MRA Technique}

Conventional whole-spine MR imaging was performed on a $1.5 \mathrm{~T}$ scanner (Twinspeed [GE Healthcare, Milwaukee, Wisconsin] and Intera Achieva [Philips Healthcare, Best, the Netherlands]) by using dedicated 8-channel and 5-channel spinal coils for the TRMRA and FP-MRA groups, respectively, with the patient in the supine position. The sequences included standard sagittal and axial T2WI, sagittal T1WI, and postcontrast sagittal and axial T1WI. Routine measures to minimize degree of motion were used for MR imaging and MRA, such as patient instruction/education, stabilization in the scanner, and sedation if required.

TR-MRA was performed by using the vendor-provided timeresolved imaging of contrast kinetics, or TRICKS, sequence. Sagittal plane was selected on 3 plane localizers with FOV of $30 \mathrm{~cm}$ (craniocaudal) extending approximately from T4 to L4 vertebral levels. The selection of FOV was based on the clinical/radiologic suspicion of the location of fistula. After acquisition of a mask, intravenous injection of $10 \mathrm{~mL}$ of gadolinium-based contrast agent (gadobutrol [Gadavist; Bayer Schering Pharma, Berlin, Germany]) was performed at $1.5 \mathrm{~mL} / \mathrm{s}$ injection rate by using a 2-cylinder MR compatible injector (Spectris; MedRad, Indianola, Pennsylvania) followed by $25-\mathrm{mL}$ saline bolus. Manual triggering was performed with no delay. Three-dimensional acquisition was performed with $320 \times 160$ matrix and $1.4 \times 1.2 \mathrm{~mm}$ in-plane resolution with $3-\mathrm{mm}$ section thickness. Parameters of $\mathrm{TR}=$ $3.5-4 \mathrm{~ms}, \mathrm{TE}=1-1.5 \mathrm{~ms}$, flip angle $=35^{\circ}, \mathrm{NEX}=0.5$, and no section gap were used. Twenty phases were obtained with 24 scan locations in each phase. The total scan time was 54 seconds, with temporal resolution of 2.2 seconds. Automated postprocessing produced background subtracted image sets for each of the 20 phases with multiplanar and cine MIPs.

FP-MRA was performed by using a manually triggered timedrun technique. Sagittal plane was selected on 3 plane localizers with FOV of $33 \mathrm{~cm}$ extending approximately from T3 to L4 vertebral levels. The selection of FOV was based on the clinical/radiologic suspicion of the location of fistula. Acquisition delay time was determined by using a $2-\mathrm{mL}$ intravenous contrast test bolus and measuring peak enhancement of the abdominal aorta using MR fluoroscopy. This was followed by intravenous administration of $18 \mathrm{~mL}$ of contrast agent at $2 \mathrm{~mL} / \mathrm{s}$ injection rate by using a 2-cylinder MR compatible injector (Spectris) followed by $20-\mathrm{mL}$ saline bolus. Gadolinium-based contrast agent, including Omnis- can (gadodiamide; GE Healthcare) or more recently MultiHance (gadobenate dimeglumine; Bracco Diagnostics, Princeton, New Jersey), was used. Single-phase 3D acquisition was performed with $400 \times 512$ matrix and $0.82 \times 1.08 \mathrm{~mm}$ in-plane resolution reconstructed to $0.64 \times 0.64 \mathrm{~mm}$ with $0.9-\mathrm{mm}$ section thickness. Scan parameters were $\mathrm{TR}=5.4 \mathrm{~ms}, \mathrm{TE}=1.76 \mathrm{~ms}$, flip angle $=$ $30^{\circ}, \mathrm{NEX}=1$, and overcontiguous sections with scan time of 47 seconds. Automated postprocessing generated background subtracted image sets and multiplanar MIPs.

\section{DSA Technique}

Spinal DSA examinations were performed on a dedicated biplanar neuroangiographic system via a femoral approach under general or local anesthesia. Multiple selective arterial injections with iodinated contrast agent (Omnipaque 300; GE Healthcare) were performed on the segmental arteries likely to supply an SDAVF. Magnification, oblique, or high frame rate angiography was used where appropriate. The angiographers first targeted the arteries expected to be supplying a fistula based on the MRA findings. Once the fistula was identified, further bilateral injections to include at least 2 vertebral levels above and below the identified fistula were performed. If the fistula was not identified at the anticipated level or MRA was negative for SDAVF, complete spinal angiography was performed.

\section{Imaging Analysis}

MR imaging and MRA images were reviewed independently by 2 experienced neuroradiologists (S.P.S. and A.B.) with 13 and 7 years of experience, respectively, who were blinded to the DSA findings.

On the MRA study, the observers made positive or negative diagnosis of SDAVF and noted the level and side of fistula if present. The observers also noted the useful signs in localization of SDAVF on MRA.

In cases where fistulas were found to be located outside the FOV, the observation of "inability to localize fistula in the MRA study" and "suspicion of fistula lower than the FOV" by the readers was considered as correct reading for the purpose of analysis. If the location of fistula was suspected at 2 levels by either of the readers, and even if 1 of the levels was correct, this ambiguity was considered an unsuccessful localization or incorrect reading. Localization within 1 vertebral level was considered as correct reading.

Information regarding the diagnosis and localization of the SDAVF was also recorded from the original clinical report of the study issued by the staff radiologist.

The readings on conventional MR images included presence or absence of intradural serpentine flow voids, T2 hyperintensity of the spinal cord, and cord enhancement.

After the readings on MR imaging and MRA studies had been completed, the observers reviewed the DSA images for presence or absence of SDAVF and noted the level and side of fistula if present. The clinical imaging reports were also retrospectively reviewed.

\section{Statistical Analysis}

The statistical measures of performance of the 2 MRA techniques and the specific signs were calculated by using DSA as the refer- 
ence standard. Sensitivity and specificity of the 2 MRA techniques were compared by using test of proportions. Cohen $\kappa$ coefficients were calculated to measure interobserver agreement. Values of $\kappa$ of $0.21-0.4,0.41-0.6,0.61-0.8$, and $0.81-1$ were considered fair, moderate, substantial, and nearly perfect, respectively. Fisher or $\chi^{2}$ test was used for categoric or dichotomous data as appropriate. Wilcoxon rank sum test was used for continuous data. Statistical significance was defined as $P<.05$. All statistical analyses were performed by using MedCalc for Windows, version 12.5 (MedCalc Software, Mariakerke, Belgium), and R, version 3.2.3 (R Foundation, http://www.r-project.org/).

\section{RESULTS}

Of 46 patients with suspected SDAVF (median age, 64 years; range, $40-89$ years; 33 [72\%] male), 15 were evaluated with TRMRA and 31 with FP-MRA. DSA identified the presence of SDAVF in 30 of $46(65 \%)$ patients, 10 of $15(67 \%)$ in the TR-MRA group and 20 of $31(64 \%)$ in the FP-MRA group. In 16 patients without evidence of SDAVF on DSA, the final diagnosis was in- fectious/inflammatory myelitis $(n=4)$, neoplasm $(n=3)$, cavernous malformation $(n=3)$, subdural hemorrhage without underlying lesion $(n=1)$, intracranial dural AVF causing myelopathy $(n=1)$, idiopathic syrinx $(n=1)$, and prominent veins/venous varix without cause identified $(n=3)$. The median time interval between MRA and DSA examinations was 5 days (interquartile range, 2-12 days). There were no significant differences between the age, sex, days between MRA and DSA, level of the fistula identified on DSA, and proportion of conventional MR imaging signs between the patients in the TR-MRA and FP-MRA groups (all $P>.05$; Table 1 ).

The observers recorded that the following signs on MRA were useful for localization of SDAVF: 1) smudge of enhancement in the region of nerve root dural sleeve connected to a branch of the segmental artery, 2) prominent intradural radicular vein, and 3) early draining radicular vein (only on TR-MRA) (Figs 1 and 2). In FP-MRA, the early draining vein sign is not useful for fistula localization because it is a single (arterial) phase technique with radicular and perimedullary veins enhancing simultaneously.

The diagnostic performance of TRMRA and FP-MRA for diagnosis of SDAVF is summarized in Table 2.

In the TR-MRA group, there was 1 false-positive diagnosis (Fig 3, Top) and no incorrect localization of SDAVF with no interobserver disagreement. In the FP-MRA group, there were 2 falsepositive diagnoses (Fig 3, Bottom), and in 2 cases, fistula was localized to more than 1 level. There were 2 instances of interobserver disagreements.

There were no significant differences in the sensitivity and specificity for diagnosis $(P>.05)$ and accuracy of localization $(P=.50)$ of SDAVF for the 2 techniques.

In 5 of $20(25 \%)$ patients in the FPMRA group, the site of the fistula iden-

Note:-IQR indicates interquartile range.
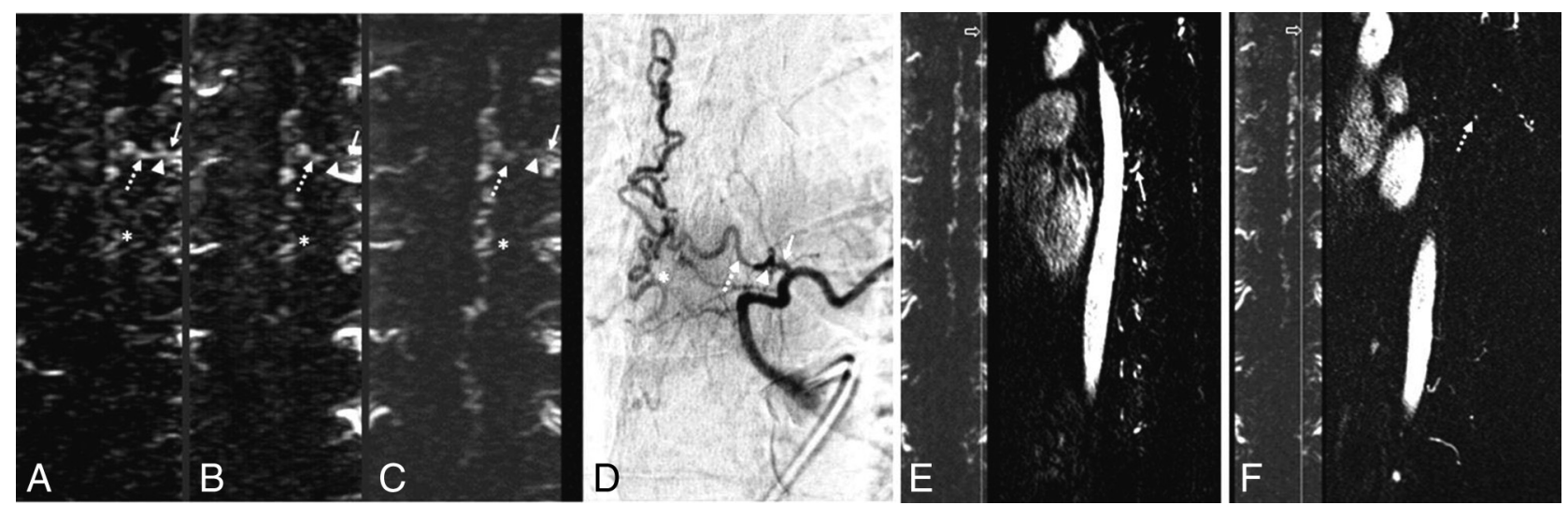

FIG 1. Imaging features of SDAVF on TR-MRA. Representative images of the coronal MIP TR-MRA images in early arterial phase (A-C) demonstrate early draining vein (dashed arrow) and smudge of enhancement at the dural sleeve (arrowhead) connected to the radiculomeningeal artery (arrow) with progressive enhancement of the arterialized perimedullary veins (asterisk). Findings can be correlated on oblique frontal projection on DSA $(D)$. $E$ and $F$ demonstrate correlation on source sagittal images; reference line on coronal images is marked by the hollow arrow. The radiculomeningeal artery at the dural sleeve (arrow, E) and prominent draining radicular vein (dashed arrow, $F$ ) are seen on sagittal images. 


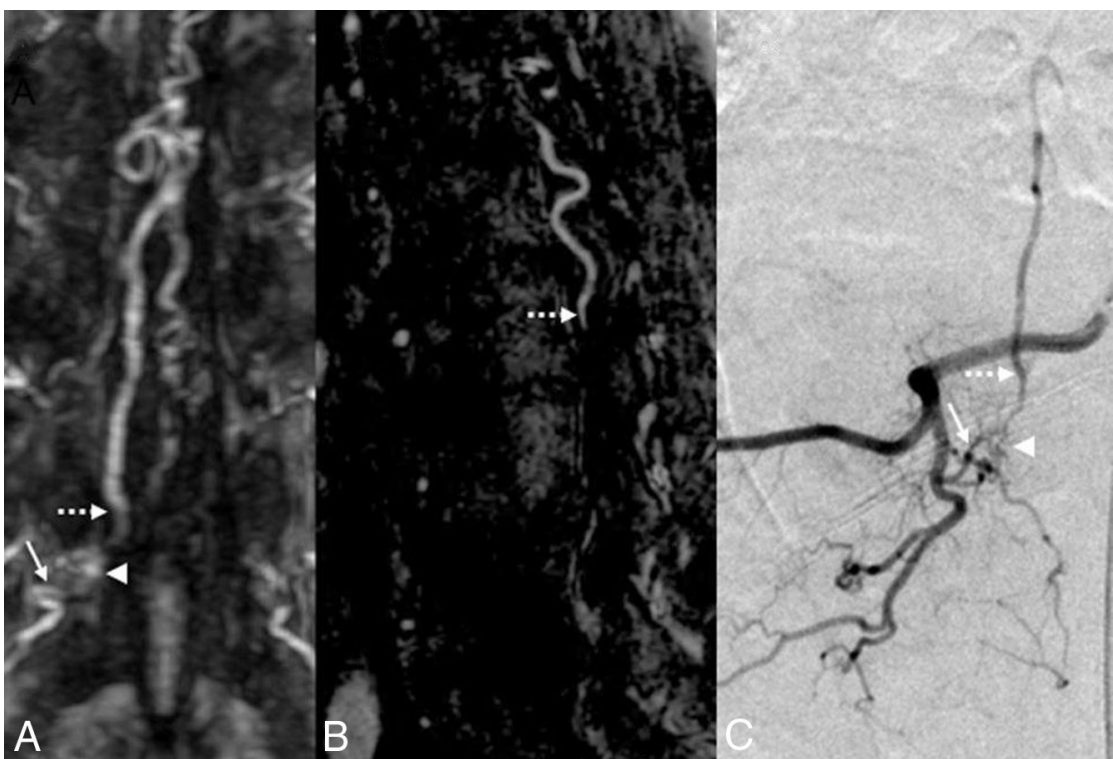

FIG 2. Imaging features of SDAVF on FP-MRA. Coronal $(A)$ and sagittal (B) MIP FP-MRA images demonstrate a prominent arterial network along the dural sleeve (arrowhead) connected to the radiculomeningeal artery (arrow), indicating the level of the fistula. A prominent radicular draining vein (dashed arrow) is noted extending superiorly from the same level. Findings correlated on oblique frontal projection DSA image (C).

Table 2: Diagnostic performance of TR-MRA and FP-MRA compared with DSA, and interobserver agreement

\begin{tabular}{lcc}
\hline & TR-MRA (95\% CI) & FP-MRA (95\% CI) \\
\hline Diagnosis of SDAVF & & \\
$\quad$ Sensitivity & $100 \%(59 \%-100 \%)$ & $100 \%(76 \%-100 \%)$ \\
Specificity & $80 \%(28 \%-99 \%)$ & $82 \%(48 \%-98 \%)$ \\
Positive predictive value & $91 \%(59 \%-100 \%)$ & $91 \%(71 \%-99 \%)$ \\
Negative predictive value & $100 \%(28 \%-100 \%)$ & $100 \%(55 \%-100 \%)$ \\
$\kappa$ Agreement & $1.0(1.0-1.0)$ & $1.0(1.0-1.0)$ \\
Localization & $100 \%(72 \%-100 \%)$ & $87 \%(62 \%-96 \%)$ \\
$\quad$ Reader accuracy to within 1 level of DSA & a \\
$\kappa$ Agreement & $1.0(1.0-1.0)$ & $0.87(0.70-1.00)$ \\
\hline
\end{tabular}

a When SDAVF location within the FOV $(n=15)$; in 5 cases where the fistula was beyond the FOV, at least 1 of the observers suspected fistula outside FOV on MRA.

tified on DSA was beyond the FOV. This was suggested on MRA by incompletely imaged prominent enhancing intradural radicular vein (Fig 4). Based on this imaging feature, both readers suspected the level of the fistula was inferior to FOV in 4 of 5 patients, whereas only 1 reader suspected the fistula was beyond the FOV in 1 patient.

No fistula level was reported in the clinical report of 1 of 10 (10\%) TR-MRA cases. In cases where the level of the fistula was described in the clinical report, there was perfect agreement between the reported level and the research readings. On the clinical reports in the FP-MRA group, the neuroradiologist did not specify the level of fistula in 5 cases, and in 1 case, fistula level was incorrectly identified. For cases where a fistula level was identified in the clinical report, there was near perfect agreement with the research reading $(\kappa=0.94)$.

\section{DISCUSSION}

DSA is the "criterion standard" for diagnosis and characterization of SDAVFs. However, it is an invasive technique that involves radiation and injection of iodinated contrast and is associated with a relatively high complication rate $(0.3 \%-2.63 \%) .{ }^{6}$ Non- selective spinal DSAs may be time-consuming and labor-intensive and may require multiple sessions to adequately localize a lesion. A major advantage of adding MRA to the imaging work-up is localization before DSA. Several studies have shown that conventional MR imaging is not helpful in fistula localization. ${ }^{7,8}$ MRA has proved useful to guide DSA and reduce the number of catheterized vessels, radiation, and contrast dose. $^{4,5}$

Contrast-enhanced MRA techniques used for evaluation of SDAVFs can be broadly classified as single-phase firstpass (or bolus-chase) technique and multiphase time-resolved technique. The selection of the technique in an institute is usually based on the preference of the neuroradiologist/neurosurgeon and feasibility. Contrast-enhanced TRMRA uses modified $k$-space sampling to provide dynamic vascular imaging with a trade-off of spatial resolution. ${ }^{9}$ In our study, the temporal resolution of TRMRA was 2.2 seconds with $1.4 \times 1.2 \mathrm{~mm}$ in-plane resolution and $3-\mathrm{mm}$ section thickness. Contrast-enhanced FP-MRA captures the arterial phase of contrast injection by manual or automated triggering at a predetermined scan delay time and provides static images with higher spatial resolution, which in our study was $0.82 \times 1.08 \mathrm{~mm}$ in-plane resolution (reconstructed to $0.64 \times 0.64$ $\mathrm{mm}$ ) with $0.9-\mathrm{mm}$ section thickness. ${ }^{4,10}$

Lim et $\mathrm{al}^{9}$ compared these techniques for evaluation of extracranial internal carotid arteries. Sandhu et $\mathrm{al}^{11}$ found better identification of perforators of the leg by using bolus-chase MRA compared with TR-MRA. Oda et $\mathrm{al}^{12}$ found that $3 \mathrm{~T}$ dynamic contrast-enhanced MRA may be more reliable compared with CTA. To the best of our knowledge, our study is the first to compare single- and multiphase spinal MRA techniques for evaluation of SDAVFs with interobserver agreement analysis.

In our study, the sensitivity and specificity of $100 \%$ and $80 \%$ of TR-MRA for detection of SDAVFs and 100\% accuracy in localization within one vertebral level is similar to previous studies. Saindane et $\mathrm{al}^{5}$ reported the sensitivity and specificity of $88 \%$ and $90 \%$, respectively, for detection of SDAVF and correct localization in 6 of 7 cases. In the study by Ali et al, ${ }^{13}$ the spinal AVFs were correctly diagnosed and localized in all 6 patients by using TRMRA. Amarouche et al ${ }^{14}$ found $98 \%$ sensitivity and $63 \%$ specificity for detection of spinal vascular malformations, with correct localization within 1 vertebral level in 39 of 47 SDAVFs.

The performance of FP-MRA in diagnosis of SDAVFs in our AJNR Am J Neuroradiol 38:206-12 Jan 2017 www.ajnr.org 

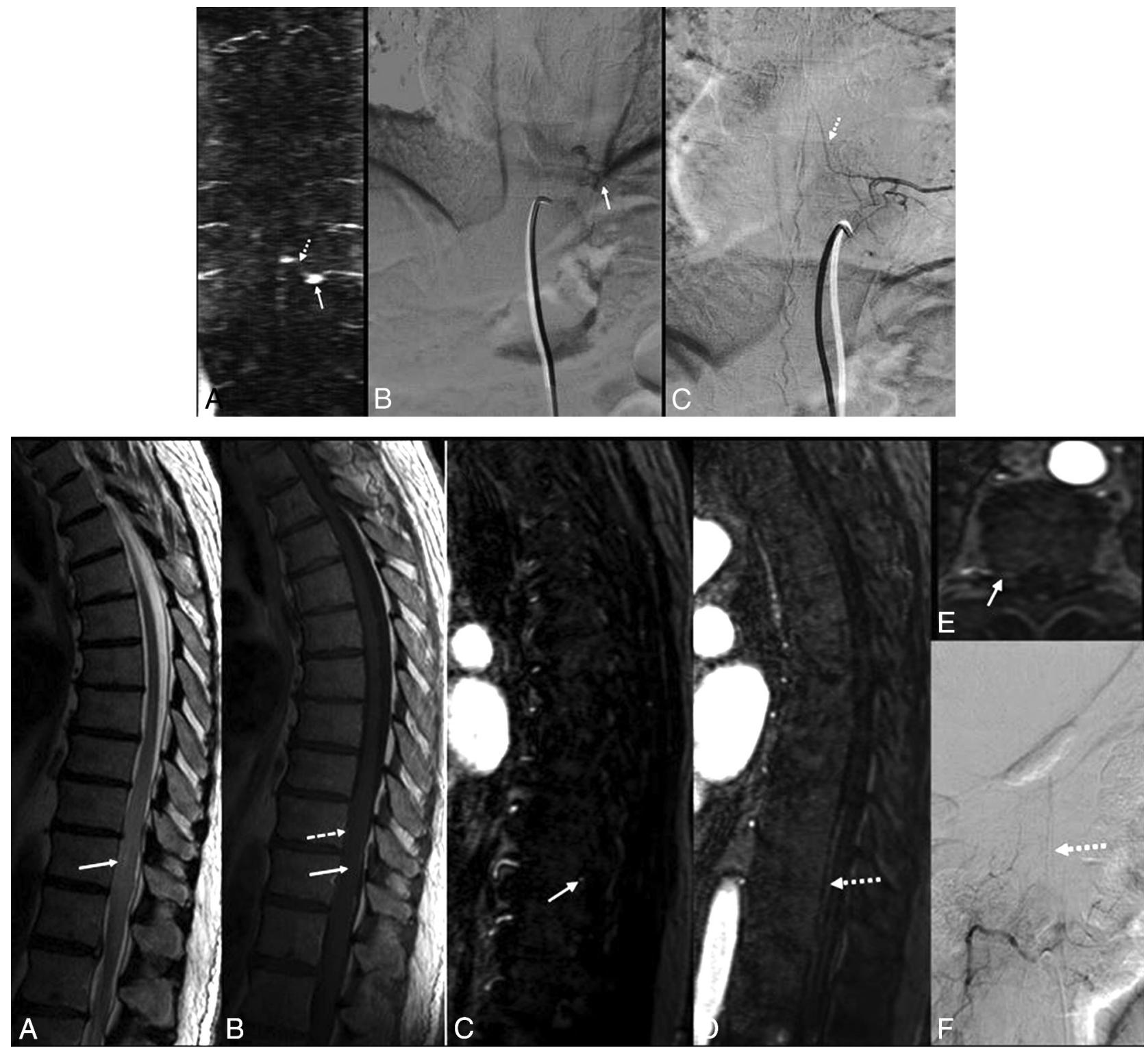

FIG 3. Top, Pitfalls in TR-MRA interpretation. Coronal MIP TR-MRA image (A) with enhancing structure at dural sleeve (arrow) mistaken for level of fistula and arterialized intradural vessel (dashed arrow) mistaken for radicular vein. On frontal projection DSA images (B and $C)$ these structures are found to be a venous varix and artery of Adamkiewicz, respectively. Bottom, Pitfalls in FP-MRA interpretation. Sagittal T2WI ( $A$ ) and enhanced sagittal TTWI $(B)$ show abnormal signal intensity (arrow, $A$ ) and mild enhancement (arrow, $B$ ) with lower thoracic cord extending to conus. There are enhancing intradural vessels in this region (dashed arrow, $B$ ). On sagittal ( $C$ and $D$ ) and axial (E) FP-MRA images, arterialized structure at dural sleeve (arrow) and arterialized intradural vessel (dashed arrow) are noted. This was found to be the artery of Adamkiewicz on DSA (F). The cord lesion was later diagnosed as astrocytoma on pathology.

study was comparable with prior studies. Saraf-Lavi et $\mathrm{al}^{8}$ found up to $100 \%$ sensitivity and $82 \%$ specificity in diagnosis. All 31 patients positive for spinal vascular lesions demonstrated enlarged perimedullary blood vessels in the study by Mull et al. ${ }^{15}$ Spinal AVF was diagnosed correctly in 20 of 22 patients in the study by Luetmer et al. ${ }^{4}$

The accuracy of lesion localization within 1 vertebral level with FP-MRA in our study (when lesion was within the FOV) was $87 \%$, which is comparable with previous studies: $81 \%$ (Lindenholz et $\mathrm{al}^{16}, n=53$ ), 73\% (Saraf-Lavi et $\mathrm{al}^{8}, n=20$ ), 100\% (Mull et al ${ }^{15}$, $n=19$ ), 93\% (Luetmer et $\mathrm{al}^{4}, n=14$ ), 100\% (Farb et al ${ }^{10}, n=9$ ), and $100 \%$ (Vargas et $\left.\mathrm{al}^{17}, n=4\right)$.

The 1 false-positive TR-MRA diagnosis of SDAVF was due to a venous varix at a neural foramen simulating smudge of enhancement and the adjacent artery of Adamkiewicz mimicking an arterialized radicular vein (Fig 3, Top). In 1 case of false-positive diagnosis in the FP-MRA group, the artery of Adamkiewicz was misinterpreted as an arterialized radicular vein (Fig 3, Bottom), and in the other case, prominent venous channels were mistaken for arterialized intradural veins on a technically limited study. In this particular case, MRA was motion degraded with some venous contamination, which was identified by diffuse epidural venous filling. No other study suffered from this technical limitation.

Although no significant difference was found between the 2 techniques in diagnosis and localization of SDAVFs in our study, there were more occurrences of uncertainty in fistula localization 

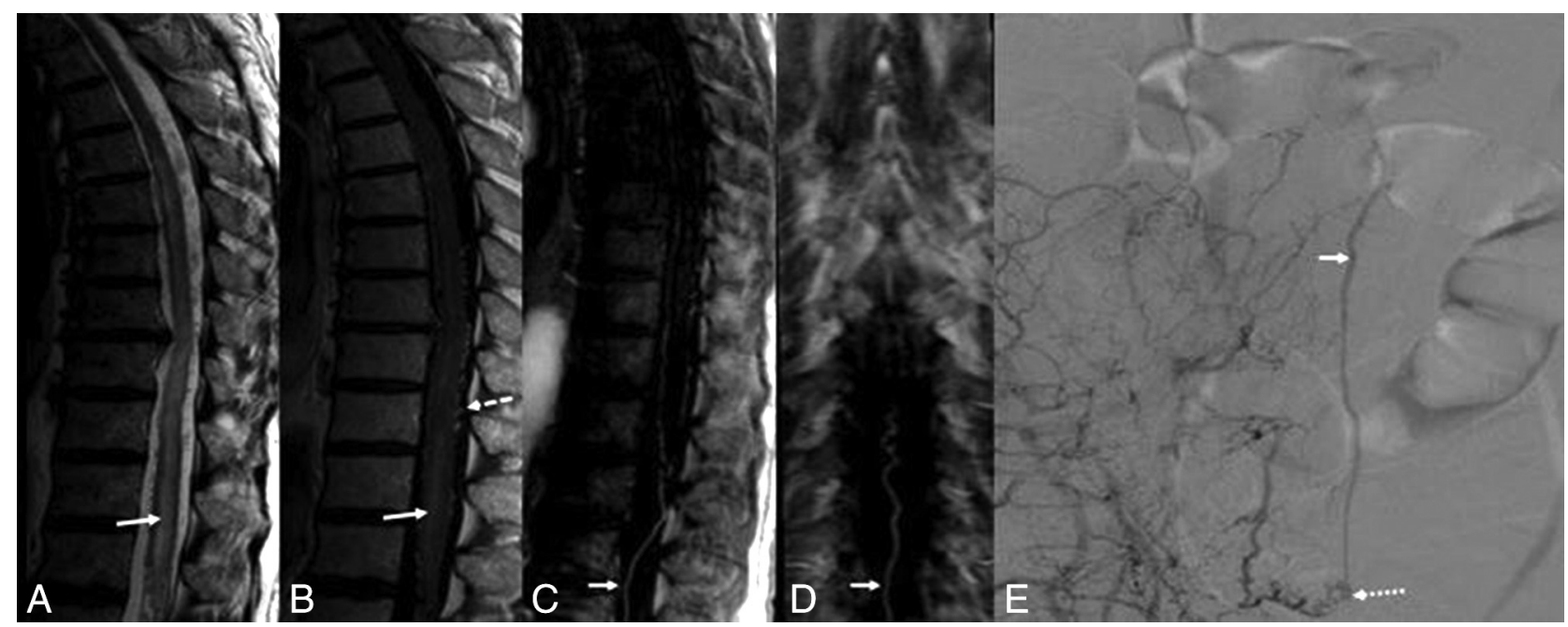

FIG 4. "Curvilinear radicular vein at the edge of the FOV" - useful sign to suspect fistula inferiorly. Sagittal T2WI ( $A$ ) and enhanced sagittal TIWI (B) show abnormal signal intensity (arrow, $A$ ) and mild enhancement (arrow, B) within lower thoracic cord extending in to conus with enhancing perimedullary vessels (dashed arrow, $C$ ). On sagittal $(C)$ and coronal $(D)$ FP-MRA images, site of fistula inferior to FOV was suspected due to the presence of prominent arterialized vessel at the lower end of the FOV (arrow). Fistula inferior to the FOV was confirmed on DSA (dashed arrow, E) with arterialized radicular vein (arrow, E).

on FP-MRA compared with TR-MRA. The ambiguity in localization in 2 cases of FP-MRA was due to a smudge of enhancement at the dural sleeve suspected by at least 1 of the observers. In the authors' opinion, the reason for this error was poor fat suppression and lack of temporal resolution on this technique. No such ambiguity was seen with TR-MRA. TR-MRA also benefits from shorter acquisition time and no requirement for contrast bolus timing.

Amarouche et $\mathrm{al}^{14}$ suggested that it would be useful to determine interobserver agreement for MRA in spinal vascular lesions. We found nearly perfect interobserver agreement of both the techniques for diagnosis and localization of SDAVFs.

The signs on MRA useful for the localization of SDAVF correspond to the angioarchitecture of these lesions. The observers found that the prominent draining radicular vein sign was difficult to identify in the mid/upper thoracic and cervical region, which correlated to the normal anatomy of the spinal venous system. The smudge of enhancement at the dural sleeve could be subtle, but connection to a radiculomeningeal artery confirmed the presence of the fistula. Lindenholz et $\mathrm{al}^{16}$ highlighted the fact that the arterial network at the dural sleeve is difficult to identify, and this could result in a gap because the vessels are too small. Although arterialized intradural veins are seen in both techniques, TR-MRA is optimal for delineation of the early draining vein due to its dynamic nature.

TR-MRA and FP-MRA are both limited by FOV, which has been reported by other investigators. ${ }^{4,5,16}$ In our study, in 5 cases in the FP-MRA group where the fistula was not covered by FOV, at least 1 of the observers suspected a lower level of fistula based on the presence of linear/curvilinear intradural radicular vein at the edge of FOV, and we believe that the presence of this sign in a suspected case of SDAVF should prompt further investigation at levels inferior to the FOV. The higher frequency of nonlocaliza- tion of fistula in clinical reports in the FP-MRA group is probably due to more fistulas located outside FOV and in the upper thorax in this group.

Limitations of our study include the small number of patients with this rare condition. The small study population results in large confidence intervals. Confirmation in a large prospective study would be ideal, however, difficult given the rarity of this condition. Future studies could also compare these 2 techniques in assessment of intracranial lesions. The retrospective design of our study may result in selection bias. Comparison may also be limited because patients in the FP-MRA and TR-MRA groups were different.

\section{CONCLUSIONS}

TR-MRA and FP-MRA were comparable in diagnosis and localization of SDAVFs and demonstrate excellent interobserver agreement, though there were more instances of ambiguity in fistula localization on first-pass MRA.

\section{REFERENCES}

1. Hurst RW, Kenyon LC, Lavi E, et al. Spinal dural arteriovenous fistula: the pathology of venous hypertensive myelopathy. Neurology 1995;45:1309-13 CrossRef Medline

2. Atkinson JL, Miller GM, Krauss WE, et al. Clinical and radiographic features of dural arteriovenous fistula, a treatable cause of myelopathy. Mayo Clin Proc 2001;76:1120-30 CrossRef Medline

3. Brinjikji W, Nasr DM, Morris JM, et al. Clinical outcomes of patients with delayed diagnosis of spinal dural arteriovenous fistulas. AJNR Am J Neuroradiol 2016;37:380-86 CrossRef Medline

4. Luetmer PH, Lane JI, Gilbertson JR, et al. Preangiographic evaluation of spinal dural arteriovenous fistulas with elliptic centric contrast-enhanced MR angiography and effect on radiation dose and volume of iodinated contrast material. AJNR Am J Neuroradiol 2005;26:711-18 Medline

5. Saindane AM, Boddu SR, Tong FC, et al. Contrast-enhanced timeresolved MRA for pre-angiographic evaluation of suspected spinal 
dural arterial venous fistulas. J Neurointerv Surg 2014;7:135-40 CrossRef Medline

6. Willinsky RA, Taylor SM, TerBrugge K, et al. Neurologic complications of cerebral angiography: prospective analysis of 2,899 procedures and review of the literature. Radiology 2003;227:522-28 CrossRef Medline

7. Geibprasert S, Pongpech S, Jiarakongmun P, et al. Cervical spine dural arteriovenous fistula presenting with congestive myelopathy of the conus. J Neurosurg Spine 2009;11:427-31 CrossRef Medline

8. Saraf-Lavi E, Bowen BC, Quencer RM, et al. Detection of spinal dural arteriovenous fistulae with MR imaging and contrast-enhanced MR angiography: sensitivity, specificity, and prediction of vertebral level. AJNR Am J Neuroradiol 2002;23:858-67 Medline

9. Lim RP, Shapiro M, Wang EY, et al. 3D time-resolved MR angiography (MRA) of the carotid arteries with time-resolved imaging with stochastic trajectories: comparison with $3 \mathrm{D}$ contrast-enhanced bolus-chase MRA and 3D time-of-flight MRA. AJNR Am J Neuroradiol 2008;29:1847-54 CrossRef Medline

10. Farb RI, Kim JK, Willinsky RA, et al. Spinal dural arteriovenous fistula localization with a technique of first-pass gadolinium-enhanced MR angiography: initial experience. Radiology 2002;222: 843-50 CrossRef Medline

11. Sandhu GS, Rezaee RP, Wright K, et al. Time-resolved and boluschase MR angiography of the leg: branching pattern analysis and identification of septocutaneous perforators. AJR Am J Roentgenol 2010;195:858-64 CrossRef Medline

12. Oda S, Utsunomiya D, Hirai T, et al. Comparison of dynamic contrast-enhanced 3T MR and 64-row multidetector $C T$ angiography for the localization of spinal dural arteriovenous fistulas. AJNR Am J Neuroradiol 2014;35:407-12 CrossRef Medline

13. Ali S, Cashen TA, Carroll TJ, et al. Time-resolved spinal MR angiography: initial clinical experience in the evaluation of spinal arteriovenous shunts. AJNR Am J Neuroradiol 2007;28:1806-10 CrossRef Medline

14. Amarouche M, Hart JL, Siddiqui A, et al. Time-resolved contrastenhanced MR angiography of spinal vascular malformations. AJNR Am J Neuroradiol 2015;36:417-22 CrossRef Medline

15. Mull M, Nijenhuis RJ, Backes WH, et al. Value and limitations of contrast-enhanced MR angiography in spinal arteriovenous malformations and dural arteriovenous fistulas. AJNR Am J Neuroradiol 2007;28:1249-58 CrossRef Medline

16. Lindenholz A, TerBrugge KG, van Dijk JMC, et al. The accuracy and utility of contrast-enhanced MR angiography for localization of spinal dural arteriovenous fistulas: the Toronto experience. Eur Radiol 2014;24:2885-94 CrossRef Medline

17. Vargas MI, Nguyen D, Viallon M, et al. Dynamic MR angiography (MRA) of spinal vascular diseases at 3T. Eur Radiol 2010;20:2491-95 CrossRef Medline 